time windows revealed a modestly elevated risk at the highest 3 exposure quintiles for exposures that occurred $>15$ years since first exposure; $\mathrm{HR}=1.28$ (95\% CI 0.90-1.82), $\mathrm{HR}=1.27(95 \%$ CI 0.93-1.73), and HR=1.27 (95\% CI 0.91-1.77), respectively; p-trend $=0.13$.

Conclusions Exposures to endotoxin with long-term, relatively intense exposures were at most weakly associated with lung cancer risk in this cohort. The findings do not support a protective effect of endotoxin, but are suggestive of possible lung cancer promotion with increasing time since first exposure.

\section{CANCER RISK ASSESSMENT IN PEOPLE HIGHLY EXPOSED TO PCBS AND PCDFS BASED ON SERUM CONCENTRATIONS 15-24 YEARS AFTER EXPOSURE}

${ }^{1,2}$ Leon Guo, ${ }^{2,3}$ Shih-Che Hsu, ${ }^{3}$ Gen-Shuh Wang. 'Environmental and Occupational Medicine, National Taiwan University College of Medicine (NTU) and NTU Hospital, Taipei, Taiwan; ${ }^{2}$ Institute of Occupational Medicine and Industrial Hygiene, College of Public Health, National Taiwan University, Taipei, Taiwan; ${ }^{3}$ Institute of Environmental Health, College of Public Health, National Taiwan University, Taipei, Taiwan

\subsection{6/oemed-2014-102362.132}

Objectives Risk associated with dioxin-like chemicals (DLCs) can be estimated using cancer slope factor (SF) derived from epidemiology data, and lifetime average daily dose (LADD). However, for shorter term exposure, such analysis has not been done. We propose a method to estimate cancer risk using internal exposure dose.

Method In 1979, approximately 2000 people in central Taiwan accidentally consumed rice oil contaminated by dioxin-like chemicals polychlorinated biphenyls (PCBs)- and dibenzofurans (PCDFs). Blood samples were collected between 1994 and 2003. Serum toxic equivalency (TEQ) was back-extrapolated to the time at the beginning of the exposure by using half-life of 8.7 years. The LADD of the background population was estimated by serum level, and the excess cancer risk of the background population was estimated by multiplying LADD by the cancer SF. Thereafter, the LADD and excess cancer risk of the Yucheng population was estimated correspondingly by the ratio of serum TEQ area under curve (AUC) between the exposed and the background population.

Results The average serum concentration of 245 exposed people in 1994-2003 and the estimated serum concentration in 1979 were $424(\mathrm{SD}=334)$ and $1602(\mathrm{SD}=1135)$ pg-TEQ/g-lipid, respectively. The estimated LADD of DLCs in background population was $2.18 \mathrm{pg}-\mathrm{TEQ} / \mathrm{kg}$-body weight/day, and the lifetime excess cancer risk caused by background exposure to DLCs is $3.4 * 10-4$. The average value of exposed people's serum TEQ AUC and the risk (i.e., 5.7*10-3) are16.8 times higher than those of the background population.

Conclusions Based on this method, individual risk can be estimated when serum concentration of DLCs are available.

\section{NON-FATAL AGRICULTURAL INJURIES: SURVEILLANCES IN THE MIDWESTERN UNITED STATES}

1,2 Lina Lander, ${ }^{1,2}$ Ketki Patel, ${ }^{1,3}$ Dana Loomis, ${ }^{1}$ Shinobu Watanabe-Galloway, ${ }^{1}$ Gleb Haynatzki, ${ }^{1}$ Rosa Gofin, ${ }^{1,2}$ Rohan Jadhav, ${ }^{1,2}$ Risto Rautiainen. ${ }^{1}$ University of Nebraska Medical Center, Omaha, Nebraska, USA; ${ }^{2}$ Central States Center for Agricultural Safety and Health, Omaha, Nebraska, USA; ${ }^{3}$ International Agency for Research on Cancer, Lyon, France

10.1136/oemed-2014-102362.133
Objectives The objective was to estimate the agriculture injury rate in in the United States. The Bureau of Labour Statistics conducts Census of Fatal Occupational Injuries and Survey of Occupational Injuries and Illness but it excludes workplaces with 10 or fewer employees or self-owned farm operations and may underestimate the agricultural injury rate.

Method The Central States Centre of Agricultural Safety and Health partnered with National Agriculture Statistics Service to annually administer agricultural injury survey. In 2012, 6953 surveys were administered to a stratified random sample of 2007 Census of agriculture respondents in seven Midwestern States. The survey included questions on demographics, type, location and source of injury, body part injured, lost work time, and cost. The data were linked to Census of agriculture for farm level attributes. Univariate and multivariate logistic regressions were used to evaluate factors associated with adult operator injuries.

Results The cumulative incidence was 60.6 injuries per 1000 farm operators. Injury incidence was significantly higher in parttime compared to full-time farmers (79.3 vs 42.6 per 1000 , p < 0.0001); farm size 1000 or more acres compared to 180-999 and $1-179$ acres (91.6 vs. 60.5 and 45.4 per $1000, \mathrm{p}=0.002$ ); at least one livestock compared to none (77.1 vs. 44.3 per 1000 , $\mathrm{p}=0.0004)$; and having a tractor with 100 or more horsepower (71.8 per $1000, \mathrm{p}=0.006$ ).

Conclusions There were substantial differences in injury incidence by individual and farm attributes. These results may be used to develop targeted interventions to reduce agricultural injuries in the Midwestern United States.

\section{JOB EXPOSURES, HEALTH BEHAVIOURS, AND WORK- RELATED INJURIES AMONG YOUNG CONSTRUCTION WORKERS IN THE UNITED STATES: A 12-YEAR FOLLOW-UP STUDY}

Xiuwen Sue Dong, Xuanwen Wang, Julie Largay. CPWR - the Center for Construction Research and Training, Silver Spring, MD, USA

\subsection{6/oemed-2014-102362.134}

Objectives Young construction workers are at increased risk for occupational injuries. This study aimed to identify factors associated with work-related injuries within this worker group in order to provide insight for injury interventions.

Method Data from nine waves (1988-2000) of the National Longitudinal Survey of Youth, 1979 cohort (NLSY79, N=12 686), were studied. Construction workers included those who worked in the construction industry for at least one wave. Job exposures were measured by frequency and types of physical efforts, number of waves worked in blue-collar jobs, and hours worked per week. Health behaviours were composed of body mass index, and dose of tobacco, alcohol, marijuana, and cocaine use. Multivariate logistic regression was employed to estimate the association between selected factors and work-related injuries after controlling for possible confounders.

Results During the 12-year follow-up period, $47 \%$ of the construction cohort experienced work-related injuries compared to $29 \%$ for their non-construction counterparts. The logistic regression results indicated that both job exposures and personal behaviours were associated with work-related injuries: blue-collar occupations (OR $=4.24,95 \%$ CI: 2.54-7.07); physical efforts $(\mathrm{OR}=1.72,95 \% \mathrm{CI}: 1.20-2.48)$; worked over $50 \mathrm{~h}$ per week $(\mathrm{OR}=1.91,95 \% \mathrm{CI}: 1.11-3.28)$; rotating/split shift $(\mathrm{OR}=2.99,95 \% \mathrm{CI}: 1.25-7.16)$; obesity $(\mathrm{OR}=1.58,95 \% \mathrm{CI}$ : 
1.04-2.41); and cocaine use on more than 10 occasions $(\mathrm{OR}=1.98$, 95\% CI: 1.31-2.99).

Conclusions The results demonstrated that construction interventions should be developed to address preventable risk factors. Young construction workers could benefit not only from enhanced work-place injury preventions, but also health behaviour interventions.

\section{TRANSITIONS BETWEEN UPPER EXTREMITY MUSCULOSKELETAL SYMPTOMS AND WORK LIMITATION OUTCOMES: A PROSPECTIVE STUDY}

'Bethany Gardner, ${ }^{1}$ Ann Marie Dale, ${ }^{2}$ Alexis Descatha, 'Bradley Evanoff. 'Washington University in St. Louis, St. Louis, MO, USA; ${ }^{2}$ Université Versailles-Saint Quentin, Versailles, France

\subsection{6/oemed-2014-102362.135}

Objectives Selection of appropriate outcome measures in studies of work-related musculoskeletal disorders (MSDs) directly affects the observed exposure-response relationship. Considering that many different factors might affect different stages of disease severity, we examined disability outcomes that represent a spectrum of disease in a newly hired working population and described the transitions between various states of symptoms and disability. Method From July 2004 to October 2006, 1107 newly hired workers were recruited to participate in the study. Subjects completed self-reported questionnaires including demographics, medical and work history, and current symptom and work status, nerve conduction studies, and a physical exam. Surveys were repeated at 6,18 , and 36 month follow-up; 827 subjects $(75 \%)$ completed all follow-ups and were included in the analysis. The outcomes of interest were presence of upper extremity symptoms and limitations in work abilities, productivity, job restrictions, lost time, and job changes due to these symptoms.

Results A majority of workers (72\%) reported symptoms at least once during the study, yet less than half $(44-46 \%)$ reported symptoms within any single follow-up period. Similarly, 31\% of workers reported work limitations due to their symptoms at least once during the study, but only $15-16 \%$ within any single follow-up period.

Conclusions These results provide evidence for the dynamic nature of both MSD symptoms and work abilities over time, which has been theorised but with few explicit studies. If the risk factors for these outcomes differ, this may explain some of the lack of clarity in the current literature on work-related risk factors and MSD.

\section{THE LIFETIME RISK APPROACH TO ESTIMATING THE BURDEN OF OCCUPATIONAL CANCER}

${ }^{1}$ Lin Fritschi, 'Renee Carey, 'Susan Peters, ${ }^{1}$ Alison Reid, ${ }^{2}$ Tim Driscoll, ${ }^{3}$ Lesley Rushton, ${ }^{4}$ Deborah Glass, ${ }^{3}$ Sally Hutchings. 'The University of Western Australia, Perth, Australia; ${ }^{2}$ Sydney University, Sydney, Australia; ${ }^{3} /$ mperial College, London, UK; ${ }^{4}$ Monash University, Melbourne, Australia

\subsection{6/oemed-2014-102362.136}

Objectives The main approaches to estimating the burden of occupational cancer are attributable risk and lifetime risk. In this presentation we will explain why we used the lifetime risk approach.

Method The lifetime risk of cancer is an estimation of an individual's risk of being diagnosed with cancer during their life (without considering occupational exposures). The lifetime risk for the general population $\left(\mathrm{LR}_{\mathrm{GP}}\right)$ is estimated by multiplying cohort person-years-at-risk (from life table data) by age-sex specific incidence rates.

The excess lifetime risk of cancer in a cohort of workers exposed to the carcinogen of interest ( $\left.\mathrm{LR}_{\text {exposed }}\right)$ is a product of the $\mathrm{LR}_{\mathrm{GP}}$ and the excess relative risk of developing cancer associated with that exposure. $\mathrm{LR}_{\text {exposed }}$ is multiplied by the prevalence of exposure to obtain the number of cancers attributable to the exposure in the general working population.

Results The lifetime risk approach estimates the number of cancers which would occur over a number of years in the future, due to exposures in a specific year. In contrast, the attributable risk approach estimates the number of cancers which would occur in a specific year due to exposures over a number of years in the past. Because we had exposure prevalence information for a specific year based on a national survey, we determined that the lifetime risk approach was more applicable in our case.

Conclusions The lifetime risk approach is an alternative method for calculating burden of disease when exposure prevalence information is available.

\section{OBTAINING POPULATION ESTIMATES OF THE PREVALENCE OF OCCUPATIONAL EXPOSURE}

${ }^{1}$ Lin Fritschi, ${ }^{1}$ Renee Carey, 'Susan Peters, 'Alison Reid, ${ }^{2}$ Deborah Glass, ${ }^{2}$ Geza Benke, ${ }^{3}$ Tim Driscoll. ${ }^{1}$ The University of Western Australia, Perth, Australia; ${ }^{2}$ Monash University, Melbourne, Australia; ${ }^{3}$ Sydney University, Sydney, Australia

\subsection{6/oemed-2014-102362.137}

Objectives Good occupational health policy requires an overall understanding of the proportion of the working population who are exposed to hazards at work. This is difficult to estimate when nearly three-quarters of the workforce are in small and medium sized companies and so not easily surveyed or monitored. We are undertaking a series of national surveys of the workforce to estimate how many people are exposed to hazards, where those people work, and to identify areas where controls could be used more effectively.

Method A random sample of the working population were invited to participate in a telephone interview regarding carcinogens at work using a web-based application (OccIDEAS). Participants were asked about their job tasks and predefined algorithms were used to automatically assign exposures.

Results Overall, $40.3 \%$ of the working population were estimated to be exposed to at least one of the 38 carcinogens we were interested in. Farmers, heavy vehicle drivers and miners were the most likely to be exposed. The most common exposures were solar radiation, diesel engine exhust and environmental tobacco smoke. We are now undertaking similar surveys to estimate the prevalence of occupational exposure to asthmagens, noise and ototoxic chemicals. We are also examining whether migrant workers are more likely to be exposed than the Australian born population.

Conclusions This study demonstrates a practical, web-based approach to collecting population information on occupational exposure prevalence.

\section{CANCER MORBIDITY AND MORTALITY OF INORGANIC LEAD EXPOSED WORKERS IN KOREA}

${ }^{1}$ Yeon-soon Ahn, ${ }^{2}$ Min-gi Kim. 'Dongguk University Ilsan Hospital, Goyang, Republic of Korea; ${ }^{2}$ Dongguk University Gyeongju Hospital, Gyeongju, Republic of Korea

10.1136/oemed-2014-102362.138 